\title{
Fatores associados à não adesão à vacina contra HPV entre estudantes de ciências da saúde
}

\section{Factors associated with non-adherence to HPV vaccine among health science students}

\author{
Amanda de Oliveira Guimarães ${ }^{1^{*}}$ \\ Orcid: https://orcid.org/0000-0001-5338-8756
}

Bianca Santos de Toledo ${ }^{3 * * *}$

Orcid: https://orcid.org/0000-0001-5157-1450

Charlise Fortunato Pedroso ${ }^{\text {******}}$

Orcid: https://orcid.org/0000-0001-6136-6485

Ingrid Aline de Jesus Goncalves ${ }^{7 * * * * * *}$

Orcid: https://orcid.org/0000-0002-1614-4119

Raquel Silva Pinheiro ${ }^{9^{* * * * *}}$

Orcid: https://orcid.org/0000-0002-4581-584X

\author{
Alice Nascimento dos Santos ${ }^{2 * *}$ \\ Orcid: https://orcid.org/0000-0002-0417-4097 \\ Marcelo Lopes de Carvalho ${ }^{4 * * * *}$ \\ Orcid: https://orcid.org/0000-0002-6897-0834
}

Thays Angelica de Pinho Santos ${ }^{6^{* * * * * *}}$

Orcid: https://orcid.org/0000-0002-6465-3356

\author{
Hellen da Silva Cintra de Paula ${ }^{8^{* \ldots * * * *}}$ \\ Orcid: https://orcid.org/0000-0003-2083-3651
}

Lyriane Apolinário de Araújo ${ }^{10^{* * * * * *}}$

\begin{abstract}
Resumo
Introdução: Adolescentes e adultos jovens são considerados grupos vulneráveis a infecção pelo papilomavírus humano (HPV), devido a comportamentos sexuais de risco como maior número de parcerias e uso inconsistente do preservativo. No Brasil a vacina contra o HPV está disponível desde 2014, contudo a adesão ainda está abaixo do esperado. Objetivos: Identificar os fatores associados à não adesão à vacina, estado vacinal e conhecimento sobre o HPV entre estudantes de ciências da saúde em Goiânia. Materiais e Métodos: Estudo transversal, realizado com 131 participantes. Foi aplicado um questionário contendo questões sobre características sociodemográficas, conhecimento do HPV e estado vacinal. Regressão de Poisson foi realizada para identificar os preditores da não adesão a vacinação. Resultados: A maioria dos estudantes era do sexo feminino (81,7\%), com média de idade de 17 anos. O escore de conhecimento sobre o HPV foi de 67,4\%. Do total, 59 (45,0\%) declararam-se vacinados. Os fatores associados à não adesão à vacina contra o HPV foram idade superior a 18 anos, sexo masculino e pais que tinham no máximo oito anos de estudo formal. Conclusão: Os achados deste estudo reforçam a necessidade de ações intersetoriais para promoção da vacinação, envolvendo as instituições de ensino e unidades de saúde, a fim de proporcionar um diálogo entre estudantes, pais, profissionais de saúde e docentes. Acredita-se que o acesso à informação qualificada possa contribuir para a mudança de comportamento e melhoria da tomada de decisão, levando os estudantes a refletirem sobre suas atitudes e riscos inerentes à infecção pelo HPV.
\end{abstract}

Palavras-chave: papillomaviridae; cobertura vacinal; estudantes de ciências da saúde; Brasil.

${ }^{1}$ E-mail: aamanda.oliveirag@hotmail.com

${ }^{2}$ E-mail: alicenascimentosantos22@hotmail.com

${ }^{3}$ E-mail: bianca_santos89@hotmail.com

${ }^{4}$ E-mail: marcelocarvalho-@hotmail.com

${ }^{5}$ E-mail: charlise.pedroso@ifg.edu.br

${ }^{6}$ E-mail: thays.santos@ifg.edu.br

${ }^{7}$ E-mail: ingrid.goncalves@ifg.edu.br

${ }^{8}$ E-mail: hellen.paula@ifg.edu.br

${ }^{9}$ E-mail: raquel.pinheiro@ifg.edu.br

${ }^{10}$ E-mail: lyriane.araujo@ifg.edu.br

\footnotetext{
* Universidade Federal de Goiás, Curso de Biotecnologia, Goiânia, GO, Brasil ** Técnica de enfermagem do Hospital de Urgência de Aparecida de Goiânia, GO, Brasil.

*** Autônoma, Goianira, GO, Brasil.

**** Operação de caldeira da empresa Cargill, Goiânia, GO, Brasil.

***** Instituto Federal de Educação, Ciência e Tecnologia de Goiás, Câmpus Goiânia Oeste, Goiânia, GO, Brasil.
} 


\begin{abstract}
Introduction: Adolescents and young adults are considered vulnerable groups to human papillomavirus (HPV) infection, due to risky sexual behaviors such as greater number of partnerships and inconsistent condom use. In Brazil the HPV vaccine has been available since 2014, however adherence is still below expectations. Objectives: To identify factors associated with nonadherence to the vaccine, vaccination status and knowledge about HPV among health science students in Goiânia. Materials and Methods: Cross-sectional study, carried out with 131 participants. A questionnaire was applied containing questions about sociodemographic characteristics, knowledge of HPV and vaccination status. Poisson regression was performed to identify predictors of non-adherence to vaccination. Results: Most students were female (81.7\%), with a mean age of 17 years. The HPV knowledge score was $67.4 \%$. Of the total, 59 (45.0\%) declared themselves vaccinated. The factors associated with non-adherence to the HPV vaccine were age over 18 years, male and parents who had at most eight years of formal study. Conclusion: The findings of this study reinforce the need for intersectoral actions to promote vaccination, involving educational institutions and health units, in order to provide a dialogue between students, parents, health professionals and teachers. It is believed that access to qualified information can contribute to changing behavior and improving decision making, leading students to reflect on their attitudes and risks inherent to HPV infection.
\end{abstract}

Keywords: papillomaviridae; immunization coverage; students health occupations; Brazil.

\section{Introdução}

Mundialmente, a infecção pelo papilomavírus humano (HPV) é uma das infecções sexualmente transmissíveis mais comum em ambos os sexos. Existem cerca de 200 genótipos diferentes de HPV e aproximadamente 40 deles podem infectar o trato anogenital e orofaringe, resultando em um amplo espectro de manifestações clínicas, que variam de verrugas a neoplasias malignas ${ }^{1}$.

No Brasil a vacina contra esse vírus foi incluída no Programa Nacional de Imunizações (PNI) em 2014, e atualmente está disponível para meninas entre nove a 14 anos e meninos entre 11 a 14 anos, sendo duas doses com intervalo de seis meses. A vacina oferecida pelo Sistema Único de Saúde (SUS) é a quadrivalente, que previne contra quatro tipos de HPV, 16 e 18, presentes em $70 \%$ dos casos de câncer cervical, e 6 e 11, relacionados a $90 \%$ dos casos de verrugas anogenitais ${ }^{2}$.

A vacinação nessa faixa etária proporciona uma elevada imunogenicidade que contrasta as respostas imunes observadas após a infecção natural ${ }^{2}$. Além disso, há evidências científicas de que a vacinação antes do início da vida sexual tem maior eficácia e custo-benefício, sendo essa a maneira mais efetiva de evitar milhares de casos e tipos de cânceres relacionados ao $\mathrm{HPV}^{3}$.

Adolescentes e adultos jovens são considerados grupos vulneráveis a essa infecção, devido a comportamentos sexuais de risco como maior número de parcerias e uso inconsistente do preservativo. No Brasil, pesquisa epidemiológica nacional sobre infecção pelo HPV encontrou uma taxa de prevalência de $53,6 \%$ entre adultos jovens, dos quais $35,2 \%$ são casos de alto risco para o desenvolvimento de câncer, evidenciando assim a importância da vacinação nesse grupo populacional como medida de prevenção ${ }^{4}$.

Estudos têm mostrado uma baixa cobertura vacinal contra o $\mathrm{HPV}^{5-7}$, o que pode ser atribuído ao conhecimento limitado sobre essa infecção e incertezas sobre a segurança ou eficácia da vacina ${ }^{8,9}$. Em se tratando de estudantes da área da saúde, as investigações têm evidenciado uma lacuna de conhecimento sobre esse agravo $^{10-13}$, o que pode ter impacto direto na adesão à vacina e na qualidade da assistência prestada por esses futuros profissionais.

Portanto, esse estudo tem como objetivo identificar os fatores associados à não adesão à vacina, estado vacinal e conhecimento sobre o HPV entre estudantes de ciências da saúde em Goiânia, Goiás. 


\section{Materiais e Métodos}

\section{Amostra e tipo de estudo}

Trata-se de um estudo observacional, de corte transversal e analítico, cuja amostra por conveniência foi constituída por estudantes de ciências da saúde do Instituto Federal de Educação, Ciência e Tecnologia de Goiás (IFG) do município de Goiânia. Foram recrutados 131 estudantes de ambos os sexos. Este estudo foi aprovado pelo Comitê de Ética em Pesquisa com Seres Humanos do Instituto Federal de Educação, Ciência e Tecnologia de Goiás, sob o parecer $\mathrm{n}^{\circ}$ 2.556.614, e autorizado pela Diretoria da Instituição de Ensino onde foi realizado o estudo.

\section{Delineamento da pesquisa}

A coleta de dados ocorreu no período de março a julho de 2018, nos períodos matutino, vespertino e noturno. Todos os discentes elegíveis foram abordados por um pesquisador e aqueles que aceitaram participar do estudo receberam orientações sobre os objetivos, riscos e benefícios da participação na pesquisa. O Termo de Consentimento Livre e Esclarecido (TCLE) foi lido pelos entrevistadores e assinado pelos participantes.

Os dados foram coletados por meio de um questionário autoaplicável contendo questões sobre características sociodemográficas, conhecimento do HPV, estado vacinal e possíveis fatores associados à não adesão à vacina contra o HPV.

\section{Critérios de Inclusão e Exclusão}

Foram incluídos na pesquisa os alunos regularmente matriculados nos cursos da área da saúde, na modalidade técnico integrado ao ensino médio em tempo integral em Análises Clínicas, Nutrição e Dietética, Vigilância em Saúde e curso técnico integrado em Enfermagem, na modalidade de Educação de Jovens e Adultos (EJA), que assinaram o TCLE e excluídos aqueles menores de 18 anos que não obtiveram a autorização de seus pais ou responsáveis legais.

\section{Procedimentos}

\section{Tratamento estatístico}

A variável de desfecho do estudo foi a resposta direta a questão sobre ter ou não recebido a vacina contra o HPV. A adesão à vacina foi definida valendo-se do autorrelato de cada estudante. Foram consideradas como variáveis independentes as características sociodemográficas como sexo, idade, raça, religião, escolaridade dos pais, estado civil e renda familiar. Para caracterizar o conhecimento sobre a infecção pelo HPV foram avaliadas as seguintes questões: meio de comunicação por onde recebeu as informações, formas de transmissão, causas, sinais, sintomas e atitudes preventivas.

A pontuação geral de conhecimento sobre o HPV foi calculada como a soma das respostas corretas dividida pelo número total de questões. Um bom nível de conhecimento foi considerado quando a pontuação geral foi equivalente a $70 \%$ de acertos. As opções de resposta foram "sim", "não" e "não sei". Uma pontuação de zero (0) foi atribuída para uma resposta incorreta ou marcada como "não sei" e um (1) ponto para uma resposta correta.

Os dados foram analisados usando o programa estatístico STATA, versão 14 (StataCorp LP, College Station, TX, USA). Média e desvio-padrão (DP) foram calculados para as variáveis contínuas. A prevalência de autorrelato de vacinação contra o HPV foi estimada com intervalo de confiança de $95 \%$ (IC 95\%). Os testes quiquadrado $\left(\chi^{2}\right)$ e teste exato de Fisher foram utilizados para verificar a associação entre as possíveis variáveis independentes e não adesão à vacina contra o HPV. Para o cálculo da razão de prevalência ajustada (RPaj), as variáveis que apresentaram valor de $\mathrm{p} \leq 0,10$ foram submetidas a análise de regressão de Poisson com variância robusta, tendo sido previamente testadas para a 
Guimarães AO, Santos NA, Toledo BS, Carvalho ML, Pedroso CF, Santos TAP, Goncalves IAJ, Paula HSC, Pinheiro RS, Araújo LA

ocorrência de interação. Valores de $p<0,05$ foram considerados significantes.

Foi calculada a sensibilidade, a especificidade, o valor preditivo positivo (VPP) e valor preditivo negativo (VPN) do autorrelato de vacinação em relação ao estado vacinal registrado no Sistema de Informação do Programa Nacional de Imunização (SIPNI).

\section{Resultados}

Um total de 131 indivíduos participaram da pesquisa. A média de idade dos discentes foi de $17 \pm \mathrm{DP}(9,4)$, variando entre 14 e 55 anos. Houve predomínio de mulheres $(81,7 \%)$, solteiros $(77,1 \%)$, com renda inferior a três salários-mínimos $(55,7 \%)$ e que se autodeclararam pardos/morenos $(57,2 \%)$. No que se refere à escolaridade dos pais, a maioria $(64,9 \%)$ tinha de nove a 12 anos de estudo e 78,6\% referiram possuir alguma religião. Mais de um terço cursavam Nutrição e Dietética (Tabela 1).

Tabela 1: Perfil sociodemográfico dos estudantes de ciências da saúde em Goiânia, Goiás, 2018.

\begin{tabular}{|c|c|c|}
\hline Variável & $\mathbf{N}^{0}$ & $\%$ \\
\hline \multicolumn{3}{|l|}{ Sexo } \\
\hline Masculino & 24 & 18,32 \\
\hline Feminino & 107 & 81,68 \\
\hline \multicolumn{3}{|l|}{ Idade (anos) } \\
\hline$<18$ & 73 & 55,72 \\
\hline $18-30$ & 33 & 25,19 \\
\hline $31-40$ & 14 & 10,69 \\
\hline$>40$ & 11 & 8,40 \\
\hline \multicolumn{3}{|l|}{ Raça/cor da pele autodeclarada } \\
\hline Branca & 37 & 28,24 \\
\hline Negra & 12 & 9,16 \\
\hline Parda & 75 & 57,25 \\
\hline Amarela & 4 & 3,05 \\
\hline Indígena & 1 & 0,77 \\
\hline Não declaração do respondente & 2 & 1,53 \\
\hline \multicolumn{3}{|c|}{ Escolaridade dos pais/responsáveis legais (anos) } \\
\hline$\leq 8$ & 33 & 25,20 \\
\hline $9-11$ & 54 & 41,22 \\
\hline$\geq 12$ & 31 & 23,66 \\
\hline Não declaração do respondente & 13 & 9,92 \\
\hline \multicolumn{3}{|l|}{ Estado civil } \\
\hline Casado & 26 & 19,85 \\
\hline Solteiro & 101 & 77,10 \\
\hline Separado/ divorciado & 3 & 2,29 \\
\hline Não declaração do respondente & 1 & 0,76 \\
\hline \multicolumn{3}{|l|}{ Religião } \\
\hline Nenhuma & 27 & 20,62 \\
\hline Católico & 48 & 36,64 \\
\hline Evangélico & 48 & 36,64 \\
\hline Espírita & 4 & 3,05 \\
\hline Outra & 3 & 2,29 \\
\hline Não declaração do respondente & 1 & 0,76 \\
\hline
\end{tabular}




\begin{tabular}{ccc}
\hline Variável & $\mathbf{N}^{\mathbf{0}}$ & $\mathbf{\%}$ \\
\hline Renda mensal familiar & & \\
$<\mathrm{R} \$ 954,00$ & & 9,92 \\
$\mathrm{R} \$ 1.908,00-2.862,00$ & 13 & 45,80 \\
$>\mathrm{R} \$ 2.862,00$ & 60 & 31,30 \\
Não declaração do respondente & 41 & 12,98 \\
$\quad$ Curso & 17 & \\
Análises Clínicas & & 21,37 \\
Enfermagem & 28 & 32,06 \\
Nutrição e Dietética & 42 & 33,59 \\
Vigilância em Saúde & 44 & 12,98 \\
\hline
\end{tabular}

Legenda: ${ }^{~}$ Salário mínimo no Brasil, ano base de 2018: R\$ 954,00.

A tabela 2 mostra as porcentagens de participantes que responderam a cada pergunta corretamente e incorretamente. $\mathrm{O}$ score de conhecimento sobre o HPV foi de 67,4\% (IC 95\%: 65,6-69,1). A média de acertos foi de 14,4 questões $(\mathrm{DP} \pm 5,2)$, de um total de 28. A maioria dos estudantes (74,6\%) conhecia a via sexual de transmissão do HPV; 40,3\% acreditavam que a infecção pelo HPV pode ser assintomática. Embora, 70,8\% dos estudantes soubessem que o HPV é o principal responsável pelo câncer de colo de útero e que o HPV pode afetar tanto homens quanto mulheres $(73,6 \%)$ e apenas $38,5 \%$ estavam cientes de que o HPV tem cura. No geral, $48,5 \%$ dos estudantes afirmaram que a infecção pelo HPV pode causar câncer de pênis, boca, ânus e vulva.

A maioria dos participantes $(90,8 \%)$ relatou que a vacina contra o HPV é ofertada pelo SUS, sendo recomendado duas doses (42,6\%). Entretanto, apenas $6,2 \%$ sabiam que a vacina oferecida pelo SUS é quadrivalente. Além disso, 20,6\% dos estudantes estavam cientes da faixa etária recomendada (11 a 14 anos) para receber a vacina contra o HPV em meninos.

Tabela 2: Frequência de respostas para cada um dos itens do questionário de conhecimento acerca da infecção pelo HPV

\begin{tabular}{lccc}
\hline$N^{0}$ & Itens & Total Verdadeir $\begin{array}{c}\text { Falso } \\
(\mathbf{n} / \%)\end{array}$ & $\begin{array}{c}\text { Não sabe } \\
(\mathbf{n} / \%)\end{array}$ \\
$(\mathbf{n} / \%)$
\end{tabular}

1. A infecção pelo HPV é comum entre a população (V)

$128 \quad 76(59,38) \quad 13(10,16) \quad 39$

$12942(32,58) \quad 14(10,84) \quad(30,46)$

2. Existem muitos tipos de HPV (V)

$(56,58)$

3. O HPV é uma infecção sexualmente transmissível (V)

$\begin{array}{llll}130 & 97(74,62) & 13(10,00) \quad 20\end{array}$

$(15,38)$

4. O HPV pode ser transmitido por sexo oral, vaginal ou anal (V)

$\begin{array}{lll}130 & 64(49,23) \quad 27(20,77) \quad 39\end{array}$

5. O HPV pode ser transmitido pelo contato direto das partes genitais (V)

$\begin{array}{lll}(24,62) & \end{array}$

6. Ter múltiplos parceiros sexuais é um fator de risco para o HPV (V)

$13099(76,15) \quad 7(5,38)$

24

$(18,47)$

7. O início da vida sexual em idade precoce aumenta o risco de contrair $130 \quad 82(63,08) \quad 15(11,54) \quad 33$ $\operatorname{HPV}(\mathrm{T})$

8. O HPV pode ser transmitido da mãe para filho (V)

$13049(37,69) \quad 23(17,69) \quad 58$

9. Uma pessoa pode ter HPV por muitos anos sem saber (V)

$128104(81,25) 21(16,41) \quad 3(2,34)$

10. O HPV pode ser transmitido por via respiratória (F)

$130 \quad 4(3,08) \quad 96(73,85) \quad 30$

$(23,07)$

11. O contato íntimo com roupas, toalhas e vaso sanitário pode transmitir $128 \quad 40(31,25) \quad 38(29,69) \quad 50$ 
Guimarães AO, Santos NA, Toledo BS, Carvalho ML, Pedroso CF, Santos TAP, Goncalves IAJ, Paula HSC, Pinheiro RS, Araújo LA

\begin{tabular}{|c|c|c|c|c|}
\hline Itens & Total & $\begin{array}{c}\text { Verdadeir } \\
\mathbf{0} \\
(\mathbf{n} / \%)\end{array}$ & $\begin{array}{l}\text { Falso } \\
(\mathbf{n} / \%)\end{array}$ & $\begin{array}{l}\text { Não sabe } \\
(\mathbf{n} / \%)\end{array}$ \\
\hline$\overline{\mathrm{HPV}}(\mathrm{V})$ & & & & $(39,06)$ \\
\hline 12. Tabagismo e etilismo são fatores de risco para aquisição de HPV (V) & 129 & $20(15,50)$ & $57(44,19)$ & $\begin{array}{c}52 \\
(40,31)\end{array}$ \\
\hline 13. A falta de higiene íntima é um fator de risco para contrair HPV (V) & 130 & $62(47,69)$ & $25(19,23)$ & $\begin{array}{c}43 \\
(33,08)\end{array}$ \\
\hline $\begin{array}{l}\text { 14. O uso de preservativo protege completamente contra infecção por } \\
\text { HPV (F) }\end{array}$ & 129 & $54(41,86)$ & $48(37,21)$ & $\begin{array}{c}27 \\
(20,93)\end{array}$ \\
\hline 15. Homens e mulheres podem se infectar pelo HPV (V) & 129 & $95(73,64)$ & $28(21,71)$ & $6(4,65)$ \\
\hline 16. A infecção pelo HPV pode causar verrugas nos órgãos genitais (V) & 129 & $58(44,96)$ & $12(9,30)$ & $\begin{array}{c}59 \\
(45,74)\end{array}$ \\
\hline 17. A infeção pelo HPV sempre apresentará sinais e sintomas (F) & 129 & $30(23,26)$ & $52(40,31)$ & $\begin{array}{c}47 \\
(36,43)\end{array}$ \\
\hline 18. A infecção pelo HPV tem cura (V) & 130 & $50(38,46)$ & $24(18,46)$ & $\begin{array}{c}56 \\
(43,08)\end{array}$ \\
\hline 19. Todos os tipos de HPV podem causar câncer $(F)$ & 129 & $23(17,83)$ & $46(35,66)$ & $\begin{array}{c}60 \\
(46,51)\end{array}$ \\
\hline 20. O HPV é o principal responsável pelo câncer de colo de útero (V) & 130 & $92(70,77)$ & $13(10,00)$ & $\begin{array}{c}25 \\
(19,23)\end{array}$ \\
\hline 21. O HPV pode causar câncer de pênis, boca, ânus e vulva (V) & 130 & $63(48,46)$ & $10(7,70)$ & $\begin{array}{c}57 \\
(43,84)\end{array}$ \\
\hline \multicolumn{5}{|l|}{ Vacina HPV } \\
\hline 23. A vacina disponível no Brasil previne contra quatro tipos de HPV (V) & 130 & $8(6,15)$ & $23(17,69)$ & $\begin{array}{c}99 \\
(76,16)\end{array}$ \\
\hline $\begin{array}{l}\text { 24. Indivíduos que já iniciaram a vida sexual podem ser vacinados contra } \\
\text { HPV (V) }\end{array}$ & 131 & $63(48,09)$ & $42(32,06)$ & $\begin{array}{c}26 \\
(19,85)\end{array}$ \\
\hline $\begin{array}{l}\text { 25. A vacina HPV é mais eficiente em pessoas que não iniciaram a vida } \\
\text { sexual (V) }\end{array}$ & 130 & $86(66,15)$ & $14(10,77)$ & $\begin{array}{c}30 \\
(23,08)\end{array}$ \\
\hline $\begin{array}{l}\text { 26. A vacina contra HPV é ofertada pelo SUS para meninas entre } 9 \text { a } 14 \\
\text { anos (V) }\end{array}$ & 131 & $105(80,15)$ & $10(7,63)$ & $\begin{array}{c}16 \\
(12,22)\end{array}$ \\
\hline $\begin{array}{l}\text { 27. A vacina contra HPV é ofertada pelo SUS para meninos entre } 11 \text { a } 14 \\
\text { anos (V) }\end{array}$ & 130 & $27(20,77)$ & $55(42,31)$ & $\begin{array}{c}48 \\
(36,92)\end{array}$ \\
\hline 28. São recomendadas duas doses da vacina contra HPV (V) & 129 & $55(42,64)$ & $54(41,86)$ & $\begin{array}{c}20 \\
(15,50)\end{array}$ \\
\hline
\end{tabular}

Legenda: $V=$ verdadeiro; $F=$ falso.

As principais fontes de informação sobre o HPV relatadas pelos participantes foram: "Escola" $(54,2 \%, \mathrm{n}=71)$, "Mídia (internet, $\mathrm{TV}$, rádio, jornal)" $(34,4 \%, \mathrm{n}=$
45), "Profissionais de saúde" $(8,4 \%, \mathrm{n}=$ $11)$, "Amigos" $(0,8 \%, \mathrm{n}=1)$ e "Outros" $(0,8 \%, \mathrm{n}=1)$ (Figura 1). 
Figura 1: Fontes de informação sobre o HPV

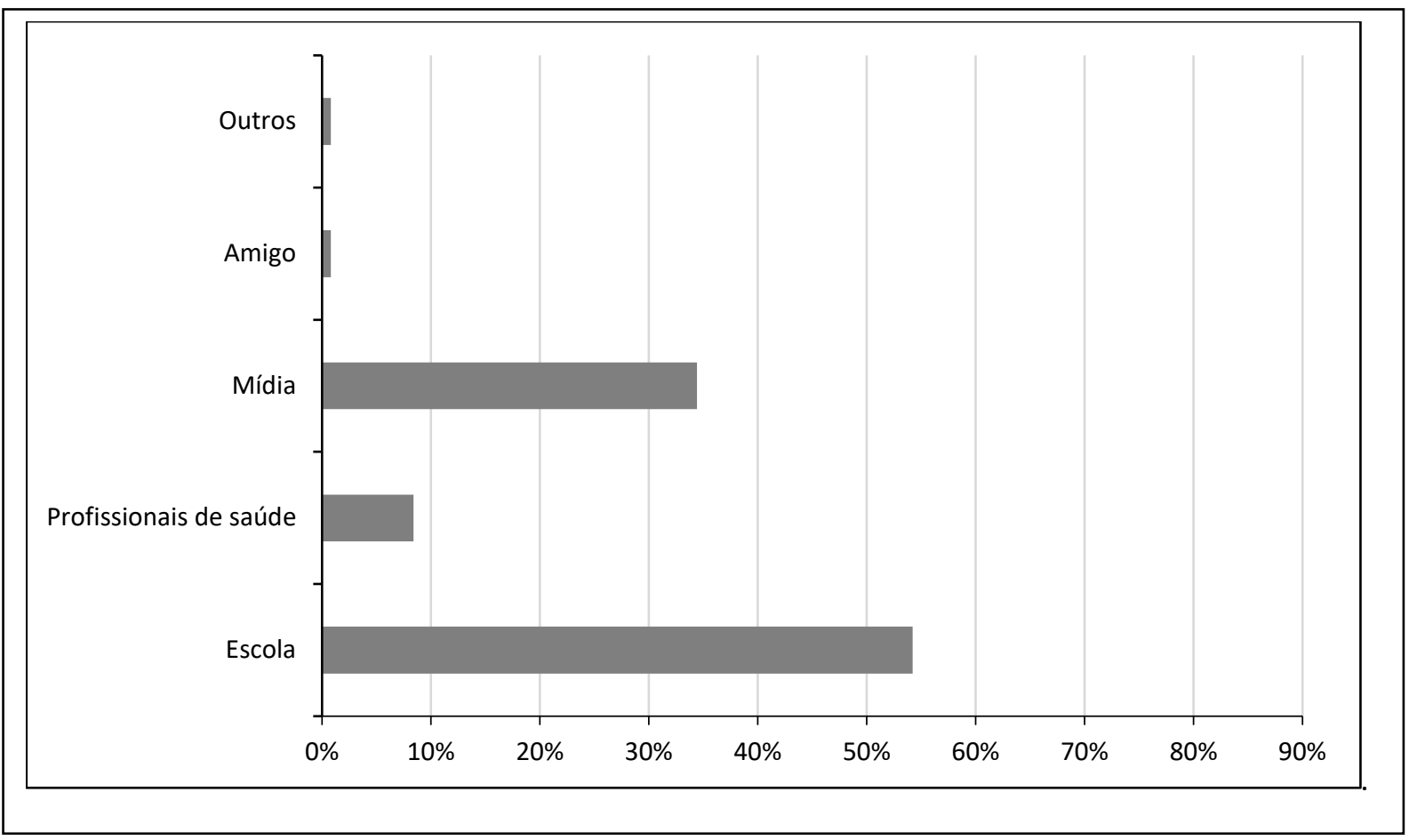

$\mathrm{Na}$ análise bivariada, as seguintes variáveis apresentaram valor de $\mathrm{p} \leq 0,10$ : sexo, idade, escolaridade dos pais, estado civil, emprego e curso (Tabela 3 ).

Tabela 3: Análise bivariada dos fatores associados à não adesão à vacina contra o HPV entre estudantes de ciências da saúde em Goiânia, Goiás, 2018

\begin{tabular}{|c|c|c|c|c|c|c|}
\hline \multirow{2}{*}{ Variáveis } & \multirow{2}{*}{ Total $^{1}$} & \multicolumn{4}{|c|}{ Vacina HPV } & \multirow[t]{2}{*}{ Valor-p } \\
\hline & & \multirow[t]{2}{*}{ Sim } & \multirow[t]{2}{*}{$\%$} & \multirow[t]{2}{*}{ Não $\%$} & $\%$ & \\
\hline \multicolumn{4}{|l|}{ Sexo } & & & \\
\hline Masculino & 24 & 2 & 8,33 & 22 & 91,67 & \\
\hline Feminino & 107 & 57 & 53,27 & 50 & 46,73 & $<\mathbf{0 , 0 0 1}$ \\
\hline \multicolumn{7}{|l|}{ Idade (anos) } \\
\hline$\leq 18$ & 87 & 58 & 66,67 & 29 & 33,33 & \\
\hline$>18$ & 44 & 1 & 2,27 & 43 & 97,73 & $<\mathbf{0 , 0 0 1}$ \\
\hline \multicolumn{7}{|l|}{ Raça/cor da pele autodeclarada } \\
\hline Branco & 37 & 15 & 40,54 & 22 & 59,46 & \\
\hline Não Branco & 93 & 44 & 47,31 & 49 & 52,69 & 0,484 \\
\hline \multicolumn{7}{|c|}{ Escolaridade dos pais/responsáveis legais (anos) } \\
\hline$\leq 8$ & 33 & 5 & 15,15 & 28 & 84,85 & \\
\hline$>8$ & 85 & 50 & 58,82 & 35 & 41,18 & $<0,001$ \\
\hline \multicolumn{7}{|l|}{ Estado civil } \\
\hline Solteiro / Divorciado / Separado & 104 & 58 & 55,77 & 46 & 44,23 & \\
\hline Casado & 26 & 1 & 3,85 & 25 & 96,15 & $<0,001$ \\
\hline \multicolumn{7}{|l|}{ Religião } \\
\hline Sim & 103 & 46 & 44,66 & 57 & 55,34 & \\
\hline Não & 27 & 13 & 48,15 & 14 & 51,85 & 0,746 \\
\hline \multicolumn{7}{|l|}{ Empregado } \\
\hline Sim & 25 & 2 & 8,00 & 23 & 92,00 & \\
\hline
\end{tabular}


Guimarães AO, Santos NA, Toledo BS, Carvalho ML, Pedroso CF, Santos TAP, Goncalves IAJ, Paula HSC, Pinheiro RS, Araújo LA

\begin{tabular}{|c|c|c|c|c|c|c|}
\hline \multirow{2}{*}{ Variáveis } & \multirow{2}{*}{ Total $^{1}$} & \multicolumn{4}{|c|}{ Vacina HPV } & \multirow[t]{2}{*}{ Valor-p } \\
\hline & & Sim & $\%$ & Não \% & & \\
\hline Não & 105 & 57 & 54,29 & 48 & 45,71 & $<0,001$ \\
\hline \multicolumn{7}{|c|}{ Renda mensal familiar ${ }^{2}$} \\
\hline$\leq \mathrm{R} \$ 954$ & 13 & 4 & 30,77 & 9 & 69,23 & \\
\hline$>\mathrm{R} \$ 954$ & 101 & 46 & 45,54 & 55 & 54,46 & 0,312 \\
\hline \multicolumn{7}{|l|}{ Curso } \\
\hline Análises clínicas & 28 & 17 & 60,71 & 11 & 39,29 & \\
\hline Enfermagem & 42 & 1 & 2,38 & 41 & 97,62 & $<0,001$ \\
\hline Nutrição e Dietética & 44 & 30 & 68,18 & 14 & 31,82 & \\
\hline Vigilância em saúde & 17 & 11 & 64,70 & 6 & 35,30 & \\
\hline \multicolumn{7}{|c|}{ Consulta médica no último ano } \\
\hline Sim & 99 & 46 & 46,46 & 53 & 53,54 & \\
\hline Não & 28 & 12 & 42,86 & 16 & 57,14 & 0,735 \\
\hline \multicolumn{7}{|c|}{ Hábito de ler/assistir jornal ou notícias sobre saúde } \\
\hline Sim & 91 & 39 & 42,86 & 52 & 57,14 & \\
\hline Não & 40 & 20 & 50,00 & 20 & 50,00 & 0,449 \\
\hline \multicolumn{7}{|c|}{ Já ouviu falar sobre a vacina contra HPV } \\
\hline Sim & 124 & 58 & 46,77 & 66 & 53,23 & \\
\hline Não & 6 & 1 & 16,67 & 5 & 83,33 & 0,220 \\
\hline
\end{tabular}

Legenda: $\quad{ }^{1}$ Número de respostas válidas. ${ }^{2}$ Salário mínimo no Brasil, ano base de 2018: R\$ 954,00.

Essas variáveis foram incluídas em um modelo de regressão de Poisson com variância robusta e após controle das variáveis de confusão, sexo, idade e escolaridade dos pais mostraram-se como fatores independentemente associados à não adesão à vacina contra o HPV (Tabela 4). A razão de prevalência para não adesão à vacina contra o HPV foi 4,60 (IC95\%: 2,82
- 7,49) vezes maior em indivíduos com idade superior a 18 anos. O modelo também apontou maior proporção de não vacinados contra o HPV em participantes do sexo masculino (RPaj: 3,04; IC95\%: 2,05 - 4,49) e cujos pais tinham no máximo oito anos de estudo formal (RPaj: 1,38; IC95\%: 1,01 $1,88)$.

Tabela 4: Análise multivariada dos fatores associados à não adesão à vacina contra o HPV entre estudantes de ciências da saúde em Goiânia, Goiás, 2018

\begin{tabular}{|c|c|c|}
\hline Variáveis & RP ajustada ${ }^{1}(\text { IC } 95 \%)^{2}$ & Valor-p \\
\hline \multicolumn{3}{|l|}{ Sexo } \\
\hline Feminino & 1,00 & \\
\hline Masculino & $3,04(2,05-4,49)$ & $<\mathbf{0 , 0 0 1}$ \\
\hline \multicolumn{3}{|c|}{ Idade (anos) } \\
\hline$\leq 18$ & 1,00 & \\
\hline$>18$ & $4,60(2,82-7,49)$ & $<\mathbf{0 , 0 0 1}$ \\
\hline \multicolumn{3}{|c|}{ Escolaridade dos pais/responsáveis legais (anos) } \\
\hline$>8$ & 1,00 & \\
\hline$\leq 8$ & $1,38(1,01-1,88)$ & 0,045 \\
\hline
\end{tabular}

Legenda: ${ }^{1}$ RP: Razão de prevalência. ${ }^{2}$ IC 95\%: intervalo de 95\% de confiança.

A sensibilidade e especificidade do autorrelato de vacinação quando comparados aos registros de imunização no SIPNI foram de $97,0 \%$ e $75,5 \%$, respectivamente. O VPP foi de $61,0 \%$ e o VPN de $98,6 \%$. De todos os participantes, $59 \quad(45,0 \%$; $\quad$ IC $\quad 95 \%$ : 36,7-53,6) 
autorrelataram a vacinação contra o HPV (dados não mostrados em tabela).

\section{Discussão}

A amostra deste estudo foi composta, predominantemente, de indivíduos jovens, do sexo feminino, solteiros, pardos e de baixa renda, sendo essas características semelhantes à de estudantes de outras regiões do Brasil ${ }^{14}$.

Esta pesquisa, assim como outras têm mostrado que o conhecimento insuficiente sobre infecção pelo HPV é um achado comum entre futuros profissionais da área da saúde ${ }^{10-13}$. Quanto menor o nível de conhecimento maior é a chance de não ser vacinado ${ }^{9,10}$. Similar ao que foi mostrado em nosso estudo, BiselliMonteiro et al. ${ }^{13}$ encontraram que embora grande parte dos discentes soubesse sobre a relação entre infecção pelo HPV e câncer de colo de útero, a maioria foi incapaz de identificar a associação entre esse patógeno e outros cânceres, como vulvar, anal, oral e peniano. Da mesma forma, poucos estudantes conheciam que existe cura para essa infecção, e nesse contexto, é importante investigar o grau de conhecimento dos discentes da área da saúde, a fim de viabilizar a implantação na grade curricular de disciplinas que abordem ações de promoção, prevenção, diagnóstico e tratamento desse agravo ${ }^{15}$.

Outras investigações também identificaram como principais fontes de informação a escola e mídia ${ }^{16,17}$. Isso pode ser atribuído não apenas à exposição formal a informações relacionadas ao HPV como parte do currículo dos cursos, mas também à aquisição de informações por meio de TV e internet.

Vale ressaltar que no Brasil, a estratégia de vacinação inicial foi resultado de uma parceria entre unidades de saúde e escolas públicas e privadas ${ }^{2}$. Paralelamente, foi realizada campanha publicitária para orientar a população sobre a importância da prevenção contra o câncer cervical em TV, rádios, jornais e redes sociais ${ }^{18}$, o que pode ter contribuído para que essas fontes de informações fossem as mais citadas.

Mundialmente, uma ampla variação das taxas de vacinação tem sido observada entre estudantes. Nesse estudo, a taxa de vacinação contra o HPV foi de $45,0 \%$ (IC 95\%: 36,7-53,6) seis vezes superior a estimada em adultos jovens brasileiros (7,5\%; IC 95\%: 6,7-8,3) e duas vezes maior a encontrada em estudantes de medicina de Brasília (21,1\%; IC 95\%: 17,2$25,4)^{7}$ e São Paulo (18,5\%; IC 95\%: 15,0$22,0)^{10}$.

O conhecimento insuficiente sobre a infecção pelo HPV pode ter contribuído para baixa adesão à vacina, ratificando o achado por Farias et al. ${ }^{9}$. Além disso, a maioria $(52,7 \%)$ dos participantes estava fora da faixa etária alvo para vacinação. No Brasil, estudo realizado entre meninas com idade igual ou superior a 15 anos identificou uma cobertura vacinal, aproximadamente, quatro vezes menor quando comparados com aquelas que estavam dentro da faixa etária recomendada para vacinação ${ }^{19}$. Ainda, 41,9\% dos estudantes acreditavam que o uso do preservativo eliminaria completamente o risco de infecção pelo HPV, o que pode ter tornado a vacinação desnecessária como forma de prevenção.

Considerando estudos realizados em outros países, a taxa de vacinação foi similar a relatada entre adolescentes da Flórida $\quad(57,0 \% \text {; IC 95\%: 49,4-64,6 })^{20}$, estudantes do ensino médio da Califórnia $(37,8 \% \text {; IC } 95 \% \text { : } 30,5-45,6)^{21}$ e universitários em Nebraska (51,0\%; IC 95\%: 41,2-59,1) 22 .

No entanto, foi superior a estimada entre estudantes da Itália (26,7\%; IC 95\%: 25,9-27,6 ${ }^{8}$, Letônia (3,3\%; IC 95\%: 1,0$7,8)^{17}$, China $(8,8 \% \text {; IC 95\%: 5,9-12,5) })^{23}$, Índia $(6,8 \% \text {; IC 95\%: 5,3-8,5 })^{24}$, Turquia (1,5\%; IC 95\%: $1,0-2,2)^{25}$ e Hong Kong $\left(23,3 \%\right.$; IC 95\%: 19,5-27,6) ${ }^{26}$. A diferença entre as taxas de prevalência talvez possa ser explicada tendo em vista as características do país em estudo. Deve-se ressaltar que na China a vacina está disponível apenas para as mulheres ${ }^{23}$, na 
Letônia somente as meninas com 12 anos são elegíveis para a vacinação ${ }^{17}$, enquanto que na İndia ${ }^{24} \mathrm{e}$ Turquia ${ }^{25}$ a vacina não está incluída no PNI, resultando em menores taxas de vacinação.

Embora, o sexo masculino tenha sido identificado como fator de risco para não adesão à vacina contra o HPV, vale ressaltar que a frequência de participantes mulheres foi maior $(81,7 \%)$ do que de homens $(18,3 \%)$. Nessa investigação, a cobertura vacinal foi significativamente menor entre os homens $(8,3 \%)$ em comparação com as mulheres $(53,3 \%)(p<0,001)$, corroborando com os dados encontrados entre universitários em Nebraska ${ }^{22}$ e Hong $\mathrm{Kong}^{26}$. Somado a isso, estudo realizado entre universitários da Suíça evidenciou que as mulheres tiveram cinco vezes mais chances de serem vacinadas do que os homens (Razão de chances ajustada: 5,8; IC 95\%: $4,1-8,2)^{11}$.

No Brasil, somente em 2017, que os meninos de 11 a 14 anos de idade foram incluídos no $\mathrm{PNI}^{2}$. Segundo Daley et al. ${ }^{27}$, a feminização da vacina e foco na prevenção do câncer de colo uterino levaram os homens a serem incluídos mais tardiamente nos programas de vacinação, o que pode ter contribuído para taxas de adesão mais baixas nesse segmento.

Além disso, a predominância de relações tradicionais de gênero é um desafio persistente à educação masculina sobre questões relacionadas à saúde sexual e reprodutiva. Os homens mantêm uma postura mais distante e são resistentes ao comportamento preventivo. Assim, ações de saúde tendem a direcionar suas iniciativas quase predominantemente para as mulheres ${ }^{16}$.

Estudo de coorte retrospectivo realizado no Brasil, verificou que $80,3 \%$ dos tipos identificados de HPV $(16,18,6$ e 11) em indivíduos com carcinoma de pênis eram imunopreveníveis, o que enfatiza a importância da vacinação em indivíduos do sexo masculino ${ }^{28}$.

$\begin{array}{ccccr}\text { Verificou-se } & \text { que } & \text { indivíduos } & \text { com } \\ \text { idade superior } & \text { a } & 18 \text { anos foi }\end{array}$

independentemente associado à não adesão à vacina contra o HPV. Esse resultado é consistente ao encontrado por outros estudos $^{19,29}$, que identificaram menor chance de ser vacinado com o aumento da idade. Além disso, indivíduos mais velhos podem sentir menos convicção sobre a praticidade e eficácia da vacinação para si mesmos, devido a uma vida sexual mais longa. ${ }^{7}$

A associação entre escolaridade dos pais/responsáveis legais (proxy de nível socioeconômico) e não adesão à vacina contra o HPV também foi observada por Faisal-Cury et al. ${ }^{19}$. Estudo realizado na Alemanha identificou que meninas cujas mães possuíam ensino superior tinham maior probabilidade de já terem sido vacinadas quando comparados àquelas cujas mães tinham educação básica (Razão de chances: 1,5 ; IC95\% 1,0-2,3) ${ }^{30}$. De fato, indivíduos com baixo nível educacional apresentam menor conhecimento sobre a infecção pelo HPV, o que pode comprometer a adesão à vacina ${ }^{5,16}$.

Este é o primeiro estudo na região Centro-Oeste do Brasil a identificar os fatores associados à não adesão à vacina, estado vacinal e conhecimento sobre o HPV entre estudantes de ciências da saúde do ensino médio, porém apresenta algumas limitações que precisam ser abordadas. A primeira delas diz respeito ao desenho do estudo, do tipo transversal, portanto, nenhuma relação causal pode ser confirmada. Em segundo lugar, foi realizado em uma única instituição de ensino com um tamanho amostral relativamente pequeno, o que limita a generalização desses resultados para a população em geral.

\section{Conclusão}

Os fatores associados à não adesão à vacina contra $\mathrm{oHPV}$ foram idade superior a 18 anos, sexo masculino e pais que tinham no máximo oito anos de estudo formal. Além disso, o conhecimento insuficiente sobre a infecção pelo HPV pode ter 
contribuído para baixa adesão à vacina. Portanto, reforça-se a necessidade de ações intersetoriais para promoção da vacinação, envolvendo as instituições de ensino e unidades de saúde, a fim de proporcionar um diálogo entre estudantes, pais, docentes e profissionais de saúde. Acredita-se que o acesso à informação qualificada possa contribuir para a mudança de comportamento e melhoria da tomada de decisão, levando os estudantes a refletirem sobre suas atitudes e riscos inerentes à infecção pelo HPV.

\section{Referências}

1. Doorbar J, Egawa N, Griffin H, Kranjec C, Murakami I. Human papillomavirus molecular biology and disease association. Rev Med Virol. 2015 Mar;25 Suppl 1(Suppl Suppl 1):223. https://doi. org/10.1002/ rmv.1822

2. Ministério da Saúde. Informe técnico da ampliação da oferta das vacinas papilomavírus humano 6, 11, 16 e 18 (recombinante) - vacina HPV quadrivalente e meningocócica C (conjugada) [Internet]; 2018 [citado 2020 Jul 13]. Disponível em: https://portalarquivos2.saude.gov.br/images/pdf/2018/marco/14/Informe-T-cnico-HPVMENINGITE.pdf.

3. Arbyn M, Xu L. Efficacy and safety of prophylactic HPV vaccines. A Cochrane review of randomized trials. Expert Rev Vaccines. 2018 Dec;17(12):1085-1091. https://doi. org/10.1080/14760584.2018.1548282.

4. Wendland EM, Villa LL, Unger ER, Domingues CM, Benzaken AS. POP-Brazil Study Group. Prevalence of HPV infection among sexually active adolescents and young adults in Brazil: The POP-Brazil Study. Sci Rep. 2020 Mar 18;10(1):4920. https://doi. org/10.1038/s41598-020-61582-2.

5. Kops NL, Hohenberger GF, Bessel M, Correia Horvath JD, Domingues C, Kalume Maranhão AG, et al. Knowledge about HPV and vaccination among young adult men and women: Results of a national survey. Papillomavirus Res. 2019 Jun; 7:123-128. https://doi. org/10.1016/j.pvr.2019.03.003.

6. Sousa PDL, Takiuti AD, Baracat EC, Sorpreso ICE, Abreu LC. Knowledge and acceptance of HPV vaccine among adolescents, parents and health professionals: construct development for collection and database composition. J Hum Growth Dev. 2018; 28(1):5868. https://doi. org/10.7322/jhgd.143856

7. Wanderley MDS, Sobral DT, Levino LA, Marques LA, Feijó MS, Aragão NRC. Students' HPV vaccination rates are associated with demographics, sexuality, and source of advice but not level of study in medical school. Rev Inst Med Trop Sao Paulo. 2019 Dec 20;61:e70. https://doi. org/10.1590/S1678-9946201961070.

8. Cocchio S, Bertoncello C, Baldovin T, Fonzo M, Bennici SE, Buja A, et al. Awareness of HPV and drivers of HPV vaccine uptake among university students: A quantitative, crosssectional study. Health Soc Care Community. 2020 Sep;28(5):1514-1524. https://doi. org/10.1111/hsc.12974.

9. Farias CC, Jesus DV, Moraes HS, Buttenbender IF, Martins IS, Souto MG, et al. Factors related to non-compliance to HPV vaccination in Roraima-Brazil: a region with a high incidence of cervical cancer. BMC Health Serv Res. 2016 Aug 22;16(1):417. https://doi. org/10.1186/s12913-016-1677-y. 
10. Costa AS, Gomes JM, Germani ACCG, da Silva MR, Santos EFS, Soares Júnior JM, et al. Knowledge gaps and acquisition about HPV and its vaccine among Brazilian medical students. $\quad$ PLoS $\quad$ One. $2020 \quad$ Mar 19;15(3):e0230058. https://doi.org/10.1371/journal.pone.0230058.

11. Jeannot E, Viviano M, Follonier MC, Kaech C, Oberhauser N, Mpinga EK, et al. Human Papillomavirus Infection and Vaccination: Knowledge, Attitude and Perception among Undergraduate Men and Women Healthcare University Students in Switzerland. Vaccines (Basel). 2019 Sep 26;7(4):130. https://doi. org/10.3390/vaccines7040130.

12. Kasymova S, Harrison SE, Pascal C. Knowledge and Awareness of Human Papillomavirus Among College Students in South Carolina. Infect Dis (Auckl). 2019 Jan 28;12:1178633718825077. https://doi. org/10.1177/1178633718825077.

13. Biselli-Monteiro M, Ferracini AC, Sarian LO, Derchain SFM. Influence of Gender and Undergraduate Course on the Knowledge about HPV and HPV Vaccine, and Vaccination Rate among Students of a Public University. Rev Bras Ginecol Obstet. 2020 Feb;42(2):96105. https://doi. org/10.1055/s-0040-1701466.

14. Brasil. Plataforma Nilo Peçanha. Brasília: MEC / Secretaria de Educação Profissional e Tecnológica do Ministério da Educação [Internet]; 2020 [citado 2020 Ago 30]. Disponível em: https://plataformanilopecanha.mec.gov.br.

15. Abreu MNS, Soares AD, Ramos DAO, Soares FV, Nunes Filho G, Valadão AF, et al. Knowledge and perception of HPV in the population over 18 years of age in the city of Ipatinga - State of Minas Gerais, Brazil. Cien Saude Colet. 2018 Mar;23(3):849-860. https://doi. org/10.1590/1413-81232018233.00102016.

16. Osis MJ, Duarte GA, Sousa MH. Conhecimento e atitude de usuários do SUS sobre o HPV e as vacinas disponíveis no Brasil [SUS users' knowledge of and attitude to HPV virus and vaccines available in Brazil]. Rev Saude Publica. 2014 Feb;48(1):123-33. https://doi. org/10.1590/s0034-8910.2014048005026.

17. Patel H, Pčolkina K, Strazdina K, Viberga I, Sherman SM, Tincello DG, et al. Awareness of HPV infection and attitudes toward HPV vaccination among Latvian adolescents. Int $\mathbf{J}$ Gynaecol Obstet. 2017 May;137(2):138-144. https://doi. org/10.1002/ijgo.12117.

18. Quevedo JP, Inácio M, Wieczorkievicz AM, Invernizzi N. The HPV vaccination policy in Brazil: official public and media communication in the face of the emergence of controversies. R Tecnol Soc. 2016;12(24):1-26.

19. Faisal-Cury A, Levy RB, Tourinho MF, Grangeiro A, Eluf-Neto J. Vaccination coverage rates and predictors of HPV vaccination among eligible and non-eligible female adolescents at the Brazilian HPV vaccination public program. BMC Public Health. 2020 Apr 6;20(1):458. https://doi. org/10.1186/s12889-020-08561-4.

20. Gerend MA, Stephens YP, Kazmer MM, Slate EH, Reyes E. Predictors of Human Papillomavirus Vaccine Completion Among Low-Income Latina/o Adolescents. J Adolesc Health. 2019 Jun;64(6):753-762. https://doi.org/10.1016/j.jadohealth.2018.12.001.

21. Mathur MB, Mathur VS, Reichling DB. Participation in the decision to become vaccinated against human papillomavirus by California high school girls and the predictors of vaccine status. J Pediatr Health Care. 2010 Jan-Feb;24(1):14-24. https://doi. org/10.1016/j.pedhc.2008.11.004. 
22. McMann N, Trout KE. Assessing the Knowledge, Attitudes, and Practices Regarding Sexually Transmitted Infections Among College Students in a Rural Midwest Setting. J Community Health. 2020 Jun 12. https://doi. org/10.1007/s10900-020-00855-3.

23. Liu Y, Jiang X, Xu L, Di N, Jiang L, Tao X. Difference Between Medical and Nonmedical Students on Knowledge, Practice, and Attitude Towards the Human Papillomavirus Vaccine in China: a Cross-Sectional Study. J Cancer Educ. 2020 Jan 6. https://doi. org/10.1007/s13187-019-01682-4.

24. Swarnapriya K, Kavitha D, Reddy GM. Knowledge, Attitude and Practices Regarding HPV Vaccination Among Medical and Para Medical in Students, India a Cross Sectional Study. Asian Pac J Cancer Prev. 2015;16(18):8473-7. https://doi. org/10.7314/apjcp.2015.16.18.8473.

25. Cinar İO, Ozkan S, Aslan GK, Alatas E. Knowledge and Behavior of University Students toward Human Papillomavirus and Vaccination. Asia Pac J Oncol Nurs. 2019 JulSep;6(3):300-307. https://doi. org/10.4103/apjon.apjon_10_19.

26. Yam PWA, Lam PL, Chan TK, Chau KW, Hsu ML, Lim YM, et al. A Cross Sectional Study on Knowledge, Attitude and Practice Related to Human Papillomavirus Vaccination for Cervical Cancer Prevention Between Medical and Non-Medical Students in Hong Kong. Asian Pac J Cancer Prev. 2017 Jun 25;18(6):1689-1695. https://doi. org/10.22034/APJCP.2017.18.6.1689.

27. Daley EM, Vamos CA, Thompson EL, Zimet GD, Rosberger Z, Merrell L, et al. The feminization of HPV: How science, politics, economics and gender norms shaped U.S. HPV vaccine implementation. Papillomavirus Res. 2017 Jun;3:142-148. https://doi. org/10.1016/j.pvr.2017.04.004.

28. de Araújo LA, De Paula AAP, de Paula HDSC, Ramos JEP, de Oliveira BR, De Carvalho KPA, et al. Human papillomavirus (HPV) genotype distribution in penile carcinoma: Association with clinic pathological factors. PLoS One. 2018 Jun 27;13(6):e0199557. https://doi. org/10.1371/journal.pone.0199557.

29. Thompson EL, Vamos CA, Vázquez-Otero C, Logan R, Griner S, Daley EM. Trends and predictors of HPV vaccination among U.S. College women and men. Prev Med. 2016 May;86:92-8. https://doi. org/10.1016/j.ypmed.2016.02.003.

30. Schülein S, Taylor KJ, König J, Claus M, Blettner M, Klug SJ. Factors influencing uptake of HPV vaccination among girls in Germany. BMC Public Health. 2016 Sep 20;16:995. https://doi. org/10.1186/s12889-016-3663-z.

\section{Como citar este artigo:}

Guimarães AO, Santos NA, Toledo BS, Carvalho ML, Pedroso CF, Santos TAP, Goncalves IAJ, Paula HSC, Pinheiro RS, Araújo LA. Fatores associados à não adesão à vacina contra HPV entre estudantes de ciências da saúde . Rev. Aten. Saúde. 2021; 19(68): 337-349. 